\title{
UTILIZAÇÃo DE ZEÓlita PREPARADA A PARTIR DE CINZA RESIDUÁRIA DE CARVÃo COMO ADSORVEDOR DE METAIS EM ÁGUA
}

\author{
Denise Alves Fungaro* e Magali Guilherme da Silva
}

Centro de Química e Meio Ambiente, Instituto de Pesquisas Energéticas e Nucleares, Travessa R, 400, 05508-900 São Paulo - SP

Recebido em 10/10/01; aceito em 12/4/02

\begin{abstract}
USE OF ZEOLITE FROM COAL BOTTOM ASH AS ADSORBENT OF METALS FROM WATER. Coal ashes produced in coal-fired power plant could be converted into zeolites and can be used as low-cost adsorbents for the treatment of effluents contaminated with high levels of toxic metals. The capacity of synthetic zeolites for the removal of cadmium, zinc and copper ions from aqueous solutions has been investigated under different operating conditions. Zeolite from bottom chimney showed higher removal efficiency for metals ions than zeolite from feed hopper and mixing mill. The results indicated that the treated bottom ash could be applied in environmental technology as an immobilizer of pollutants.
\end{abstract}

Keywords: synthetic zeolites; low-cost adsorbents; toxic metals.

\section{INTRODUÇÃO}

Um dos principais problemas ambientais causados por usinas termoelétricas é derivado da produção de toneladas de cinzas de carvão no processo de geração de energia elétrica. A necessidade de retirar a baixo custo as cinzas da usina fez com que se adotassem práticas de disposição em áreas inadequadas e sem as medidas de proteção necessárias.

A lixiviação de áreas de disposição de cinzas traz consigo a possibilidade de que elementos menores, como metais pesados, e íons sulfato possam ter acesso ao lençol freático contaminando fontes de abastecimento atuais e potenciais. $\mathrm{O}$ teor de compostos solúveis em cinza varia de traços a vários por cento e estas espécies também podem entrar na cadeia alimentar via plantas. O principal esforço no sentido de mitigar os impactos ambientais decorrentes da disposição destes resíduos no meio ambiente deve ser dirigido no sentido de analisar suas potencialidades para a utilização em outros processos industriais.

A maior parte do carvão consumido atualmente em usinas termoelétricas é queimada em caldeiras de carvão pulverizado. Neste tipo de caldeira, mais de três quartos da cinza produzida é leve o bastante para ser arrastada com os gases de combustão (cinza leve ou volante), sendo na sua maior parte coletada por equipamentos de retenção, como precipitadores eletrostáticos. As cinzas remanescentes são densas o suficiente para não serem emitidas para a atmosfera e caem, no fundo da caldeira, fundidas em partículas maiores (cinza pesada ou residuária).

As cinzas de carvão mineral são constituídas basicamente de sílica $\left(\mathrm{SiO}_{2}\right)$ e alumina $\left(\mathrm{Al}_{2} \mathrm{O}_{3}\right)$, que respondem por cerca de $50 \%$ e $30 \%$ da sua massa, respectivamente. Utilizando-se as propriedades das cinzas de carvão é possível convertê-las em zeólita após tratamento químico. A capacidade de troca catiônica das cinzas é $100 \mathrm{mmol} \mathrm{kg}^{-1} \mathrm{e}$ pode aumentar para mais de $3000 \mathrm{mmol} \mathrm{kg}^{-1}$ após o tratamento ${ }^{1-3}$.

A zeólita é um aluminosilicato cristalino com armação estrutural incluindo cavidades ocupadas por cátions grandes e moléculas de água, ambos tendo considerável liberdade de movimento, permitindo troca iônica e desidratação reversível ${ }^{4-6}$. A remoção das moléculas de água e a substituição dos cátions intercambiáveis não alteram a estrutura básica das zeólitas.

*e-mail: dfungaro@net.ipen.br
A utilização das zeólitas como adsorvedor de baixo custo é uma alternativa efetiva à precipitação química para a remoção de metais de águas e efluentes industriais.

A aplicação de zéolitas naturais como trocadoras de cátions para a recuperação de metais e a proteção ambiental foi descrita em artigos de revisão ${ }^{7-8}$. A remoção de diversos metais tóxicos de águas por zeólitas preparadas com cinza leve de carvão foi estudada com variação das condições experimentais ș-3,-10. $^{1-}$

As vantagens do uso da zeólita obtida a partir das cinzas de carvão são: (1) é sintetizada a partir de resíduo abundante; (2) o reagente usado na síntese pode ser reaproveitado; (3) a zeólita pode ser regenerada com NaCl; (4) o metal pode ser recuperado; (5) as resinas poliméricas trocadoras de íons disponíveis comercialmente são relativamente caras; (6) a estrutura da zeólita confere seletividade por tamanho, forma e carga; (7) estabilidade térmica e resistência à radiação.

O desempenho das zeólitas sintetizadas a partir de cinzas de carvão irá determinar se a conversão das cinzas é ou não um processo de reciclagem viável.

O objetivo deste estudo foi determinar a eficiência de zeólitas sintetizadas a partir de cinzas residuárias de carvão em remover cádmio, zinco e cobre de soluções aquosas. A otimização do processo de adsorção em relação às condições experimentais $(\mathrm{pH}$; temperatura; tempo de agitação; massa e granulometria da zeólita; concentração e tipo do analito e diferentes tipos de cinzas) foi examinada.

\section{PARTE EXPERIMENTAL}

Todas as soluções foram preparadas com reagentes de grau analítico e água desionizada Milli-Q.

\section{Preparação da zeólita e análise}

As cinzas residuárias de carvão da Usina Termoelétrica de Figueira foram utilizadas na preparação das zeólitas. O carvão provém das minas Amando Simões e do Poço 115 situados no Município de Figueira, na porção nordeste do estado do Paraná, e pertencem à Companhia Carbonífera do Cambuí.

As frações passantes na peneira $\mathrm{ABNT} \mathrm{n}^{\circ} 70(0,210 \mathrm{~mm})$ e ABNT $\mathrm{n}^{\circ} 100(0,150 \mathrm{~mm})$ foram utilizadas para a preparação das zeólitas com cinzas de diferentes locais, conforme Tabela 1. A cinza do Malachador 
tinha partículas menores que a abertura da peneira ${ }^{\circ} 100$ e foi usada conforme coletada. O procedimento de $\mathrm{Henmi}^{11}$ foi seguido no tratamento hidrotérmico das cinzas com solução de $\mathrm{NaOH}$ 3,5 M.

Tabela 1. Zeólitas preparadas a partir de diferentes cinzas

\begin{tabular}{ll}
\hline Zeólita & Cinza \\
\hline ZT/70 e ZT/100 & Tremonha - Caldeira III \\
ZM & Malachador - Caldeira II \\
ZB/70 e ZB/100 & Base da chaminé - Caldeira III \\
\hline
\end{tabular}

A composição química das cinzas foi determinada por fluorescência de raios-X (RIX-3000 RIGAKU) e os dados mostraram que os principais constituintes são os óxidos de silício (37 $44 \%)$, alumínio $(8-11 \%)$, enxofre $(2-6 \%)$ e ferro $(5-8 \%)$. Os demais óxidos encontram-se ao nível de traço. A Tabela 2 mostra a análise imediata de base seca e a relação Si/Al das amostras de cinza. A análise por difração de raios-X (RINT-2000 RIGAKU) revelou que o principal produto obtido após o tratamento hidrotérmico das diferentes cinzas era a zeólita P1 com traços de quartzo, mulita e hidroxisodalita.

Tabela 2. Análise imediata de base seca de amostras de cinzas

\begin{tabular}{cccccc}
\hline Amostra & $\begin{array}{c}\text { Umidade } \\
(\%)\end{array}$ & $\begin{array}{c}\text { Cinza } \\
(\%)\end{array}$ & $\begin{array}{c}\text { Material } \\
\text { Volátil }(\%)\end{array}$ & $\begin{array}{c}\text { Carbono } \\
\text { fixo }(\%)\end{array}$ & Si/Al \\
\hline CT & 1,82 & 67,33 & 11,6 & 21,1 & 4,38 \\
CM & 14,4 & 82,45 & 7,48 & 10,1 & 4,23 \\
CB & 1,25 & 90,29 & 5,31 & 4,40 & 3,86 \\
\hline
\end{tabular}

CT: cinza da Tremonha; CM: cinza do Malachador; $\mathrm{CB}$ : cinza da base da chaminé

\section{Estudos sobre a remoção de metais}

O estudo da remoção dos metais pelas zeólitas foi realizado utilizando-se processos descontínuos sob agitação. Uma alíquota da solução do metal $(100 \mathrm{~mL})$ com concentração conhecida foi colocada com determinada massa de zeólita. A suspensão foi agitada por um tempo. O sobrenadante foi separado por centrifugação e a concentração do metal nesta solução foi determinada por titulação complexiométrica com EDTA. Os dados das isotermas foram obtidos após um tempo de equilíbrio de $24 \mathrm{~h}$. Os estudos foram realizados utilizando-se concentrações do metal na faixa de $127-895 \mathrm{mg} \mathrm{L}^{-1}$, as quais são as concentrações usuais encontradas em águas de lavagem de indústrias de galvanoplastia ${ }^{12}$. O método foi aplicado em amostras reais de banho galvânico de zinco diluídas por um fator de 100 e as concentrações finais dos metais foram analisadas por voltametria de redissolução anódica (Autolab Basic PGSTAT 30).

\section{RESULTADOS E DISCUSSÃO}

\section{Efeito dos tipos de cinzas}

Os diferentes tipos de cinzas de carvão amostradas na termoelétrica de Figueira podem gerar zeólitas diferentes durante o tratamento hidrotérmico. A natureza da zeólita influi no processo de adsorção do metal. Cada tipo de zeólita irá apresentar composição química, capacidade de troca iônica e tamanho de poros e partículas características.

A Figura 1 apresenta os resultados da remoção do $\mathrm{Cd}, \mathrm{Zn}$ e $\mathrm{Cu}$ nos diferentes tipos de zeólita. a)

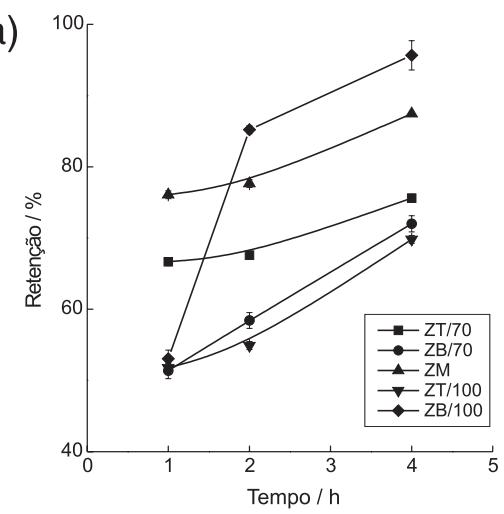

b)

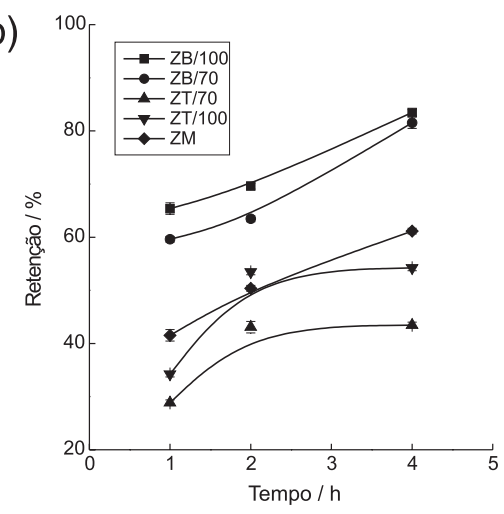

C)

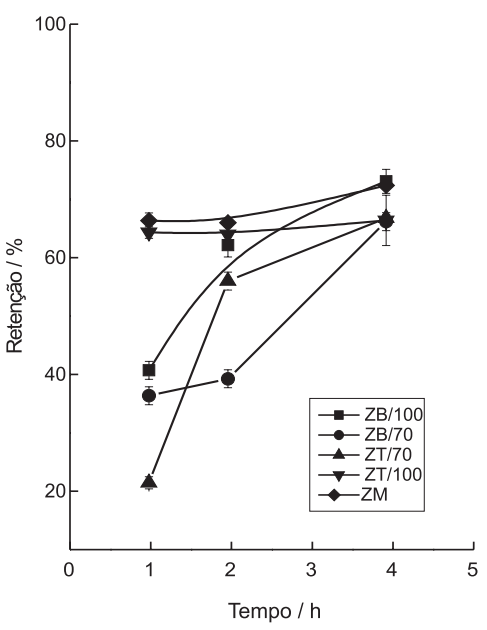

Figura 1. Porcentagem de retenção do (a) $\mathrm{Cd}^{2+}$; (b) $\mathrm{Zn}^{2+}$; (c) $\mathrm{Cu}^{2+}$ em função

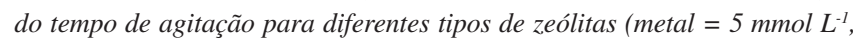
$1 \mathrm{~g}$ zeólita, $\mathrm{T}=25^{\circ} \mathrm{C}$ )

A eficiência de retenção dos metais $\mathrm{Cd}, \mathrm{Zn}$ e $\mathrm{Cu}$ aumentou com o tipo de zeólita após 4 h de agitação na seguinte ordem: $\mathrm{ZT}<\mathrm{ZM}<$ $\mathrm{ZB}$. Os resultados demonstraram claramente a superioridade da ZB/ 100 para remover os íons.

Os dados da análise imediata das amostras (Tabela 2) mostraram que a cinza da Base da chaminé provém do carvão que foi mais queimado, apresentando o maior teor de cinza e material volátil e a menor porcentagem de carbono fixo, exatamente o oposto da cinza da Tremonha. Já a cinza do Malachador apesar de constituir-se das partículas mais finas, apresenta menor teor de cinza do que a cinza da Base da chaminé.

Os resultados da fluorescência de raios-X indicaram que ZB apresentaria a menor relação $\mathrm{Si}: \mathrm{Al}$ por causa da sua cinza de origem. Geralmente, as maiores capacidades de troca são observadas em 
zeólitas que apresentam baixa relação $\mathrm{SiO}_{2} / \mathrm{Al}_{2} \mathrm{O}_{3}$, mas há exceções devido a impurezas ou variação na composição química ${ }^{5}$.

Observando-se as zeólitas que utilizaram os mesmos tipos de cinzas, aquelas com partículas mais finas $(\leq 0,15 \mathrm{~mm})$ adsorvem mais do que aquelas com partículas mais grossas, devido ao aumento da área superficial externa. O comportamento fora deste padrão é atribuído à heterogeneidade do material que ainda é observado na zeólita, mesmo após o tratamento químico.

A zeólita ZB/100 foi escolhida para o estudo de outros parâmetros que afetam a capacidade de troca do adsorvedor nos processos descontínuos.

\section{Efeito do pH inicial na remoção do metal}

O estudo do efeito do $\mathrm{pH}$ inicial da solução na capacidade de retenção da zeólita é importante porque os efluentes industriais normalmente apresentam uma grande variação no valor de $\mathrm{pH} . \mathrm{O} \mathrm{pH}$ tem um impacto significante na remoção dos metais por zeólita, já que pode influenciar o caráter dos íons trocáveis e o caráter da própria zeólita ${ }^{13}$.

No processo de troca iônica deve-se manter o pH da solução em um nível suficientemente baixo, para evitar o limite de solubilidade dos metais, e suficientemente alto, para minimizar a competição de prótons por sítios nas partículas da zeólita e a degradação da sua estrutura, com correspondente perda de espécies de alumínio. O valor de $\mathrm{pH}$ de precipitação dos hidróxidos é 8,4; 7,0 e 6,1 para o $\mathrm{Cd}$, $\mathrm{Zn}$ e $\mathrm{Cu}$, respectivamente ${ }^{14}$.

A influência do pH inicial na capacidade de troca da zeólita ZB/ 100 é apresentada na Figura 2. A maior porcentagem de remoção foi obtida em $\mathrm{pH} 4,5$ para o $\mathrm{Cd}^{2+}$ e $\mathrm{Cu}^{2+}$ e 5,0 para o $\mathrm{Zn}^{2+}$. A Figura 3 mostra a variação do $\mathrm{pH}$ final com o pH inicial das soluções dos íons metálicos. Um mecanismo de troca iônica predominante com precipitação negligenciável foi observado em $\mathrm{pH}$ inicial 2,5 a 5,0 para o $\mathrm{Cd}^{2+}$ e $\mathrm{Zn}^{2+}$, e 2,5 a 3,5 para o $\mathrm{Cu}^{2+}$.

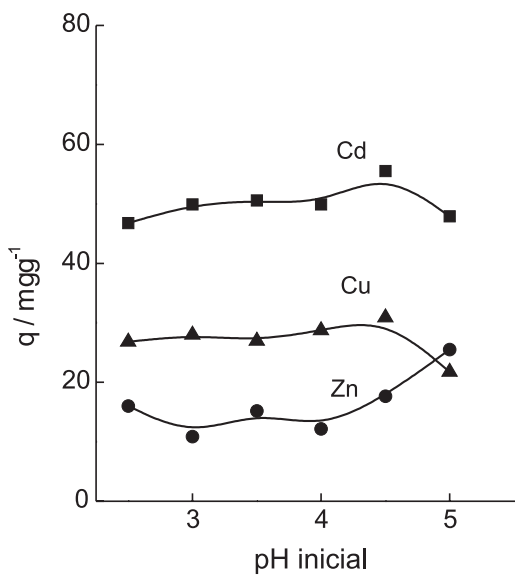

Figura 2. Efeito do pH inicial na capacidade de troca da zeólita (metal = $5 \mathrm{mmol} \mathrm{L} \mathrm{L}^{-1}, 1 \mathrm{~g}$ zeólita, $T=25^{\circ} \mathrm{C}, 2 \mathrm{~h}$ de agitação)

\section{Efeito da quantidade de zeólita}

A eficiência de remoção é dependente da concentração inicial do metal e da relação massa da zeólita por volume da solução dos metais. Quanto maior for a razão, maior será a porcentagem de remoção dos metais. No entanto, poderá ocorrer a precipitação do metal como hidróxido em conjunto com a adsorção, se houver um aumento significativo do $\mathrm{pH}$. A Figura 4 mostra o efeito da densidade da suspensão no $\mathrm{pH}$ final após $2 \mathrm{~h}$ de agitação.

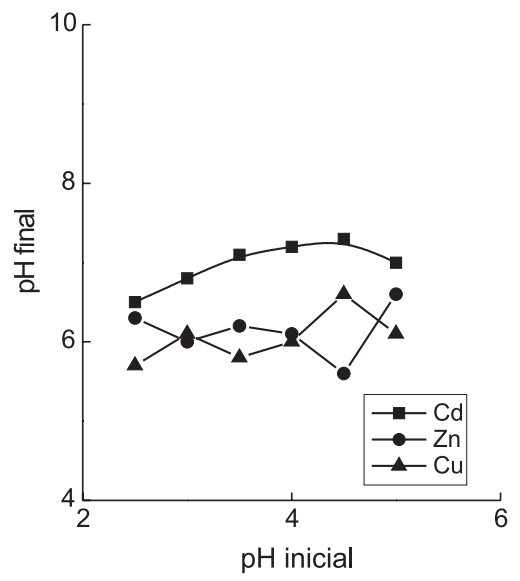

Figura 3. Dependência do pH final com o pH inicial (metal $=5 \mathrm{mmol} \mathrm{L}^{-1}, 1 \mathrm{~g}$ zeólita, $T=25^{\circ} \mathrm{C}, 2 \mathrm{~h}$ de agitação)

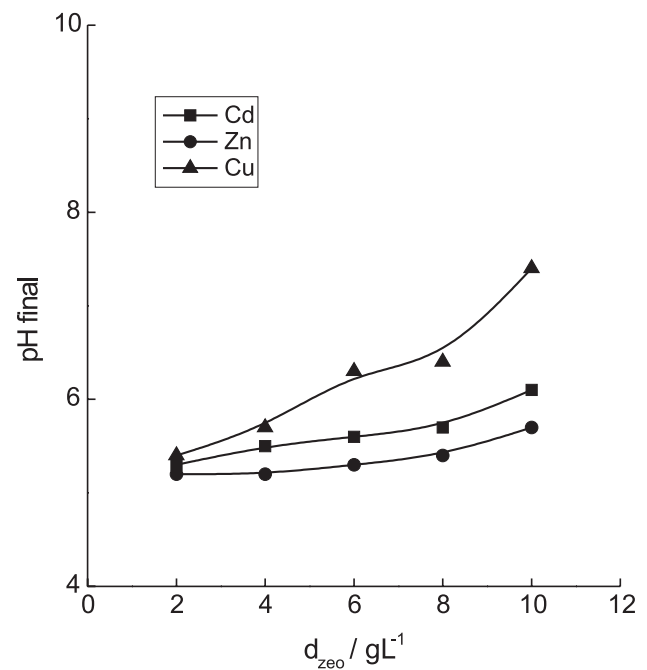

Figura 4. Efeito da densidade da suspensão no $\mathrm{pH}$ final (metal $=5 \mathrm{mmol} \mathrm{L}^{-1}$, $T=25^{\circ} \mathrm{C}, 2 \mathrm{~h}$ de agitação)

Aumentando-se a densidade da suspensão de 2 a $10 \mathrm{~g} \mathrm{~L}^{-1}$, o valor do $\mathrm{pH}$ final permaneceu bem abaixo do $\mathrm{pH}$ de precipitação do cádmio e do zinco no fim do contato metal-adsorvedor e, portanto, a precipitação foi negligenciável. No caso do $\mathrm{Cu}^{2+}$, o processo de adsorção foi predominante para densidade da suspensão $\leq 8 \mathrm{~g} \mathrm{~L}^{-1}$.

\section{Isotermas de adsorção e aplicações}

A análise dos dados da isoterma é importante para desenvolver uma equação que represente realmente os resultados e que possa ser usada para propósitos de dimensionamento de equipamentos a serem utilizados na remoção de poluentes. Existem vários modelos publicados em literatura para descrever os dados experimentais das isotermas de adsorção. Os modelos de Langmuir e Freundlich são os mais freqüentemente usados para descrever isotermas para aplicações em tratamento de águas e efluentes ${ }^{15,16}$.

A expressão linear de Langmuir é:

$\frac{C_{e}}{q_{e}}=\frac{1}{Q_{o} b}+\frac{C_{e}}{Q_{o}}$

onde $\mathrm{C}_{\mathrm{e}}$ é a concentração do metal no equilíbrio $\left(\mathrm{mg} \mathrm{L}^{-1}\right), \mathrm{q}_{\mathrm{e}}$ é a 
quantidade adsorvida no equilíbrio $\left(\mathrm{mg} \mathrm{g}^{-1}\right), \mathrm{Q}_{\mathrm{o}}\left(\mathrm{mg} \mathrm{g}^{-1}\right)$ e b $\left(\mathrm{L} \mathrm{mg}^{-1}\right)$ são constantes relacionadas com a capacidade de adsorção máxima e a energia de adsorção, respectivamente.

$\mathrm{O}$ gráfico linear de $\mathrm{C}_{\mathrm{e}} / \mathrm{q}_{\mathrm{e}}$ vs $\mathrm{C}_{\mathrm{e}}$ confirma a validade do modelo de Langmuir para o processo. A equação de reta obtida apresentará coeficiente angular correspondente a $1 / \mathrm{Q}_{\mathrm{o}}$ e coeficiente linear correspondente a $1 / \mathrm{Q}_{0}$ b. A característica essencial da isoterma pode ser expressa pela constante adimensional chamada parâmetro de equilíbrio, a qual é definida como ${ }^{17}$ :

$\mathrm{R}_{\mathrm{L}}=\frac{1}{1+b C_{o}}$

onde $\mathrm{C}_{\mathrm{o}}$ é a concentração inicial do metal mais alta $\left(\mathrm{mg} \mathrm{L}^{-1}\right)$ e b é a constante de Langmuir. $\mathrm{O}$ valor de $\mathrm{R}_{\mathrm{L}}$ entre 0 e 1 indica adsorção favorável.

A forma linear da equação de Freundlich é dada pela equação:

$\log \mathrm{q}_{\mathrm{e}}=\log \mathrm{K}_{\mathrm{f}}+\frac{1}{n} \log C_{e}$

onde $\mathrm{K}_{\mathrm{f}}\left(\mathrm{mg} \mathrm{g}^{-1}\right)$ e $\mathrm{n}$ são constantes relacionadas com a capacidade de adsorção e a intensidade de adsorção, respectivamente. Os valores de $K_{f}$ e $n$ podem ser obtidos pela intersecção e inclinação do gráfico linear de $\log \mathrm{q}_{\mathrm{e}}$ versus $\log \mathrm{C}_{\mathrm{e}}$. $\mathrm{O}$ valor de $\mathrm{n}$ entre 2 e 10 indica processo de adsorção favorável.

As isotermas de adsorção de Langmuir e Freundlich do $\mathrm{Cd}^{2+}$, $\mathrm{Zn}^{2+}$ e o $\mathrm{Cu}^{2+}$ obtidas a $25^{\circ} \mathrm{C}$ são mostradas nas Figuras 5 e 6.

Os parâmetros de adsorção encontrados estão listados na Tabela 3. Os valores experimentais dos coeficientes de correlação das retas mostraram que ambos os modelos de isotermas de Langmuir e Freundlich podem adequadamente descrever os dados de adsorção.

Tabela 3. Parâmetros dos modelos de isoterma de Freundlich e Langmuir para os vários metais e zeólita ZB/100

\begin{tabular}{lccccc}
\hline Metal & \multicolumn{2}{c}{ Freundlich } & \multicolumn{3}{c}{ Langmuir } \\
& $\mathrm{K}_{\mathrm{f}}\left(\mathrm{mg} \mathrm{g}^{-1}\right)$ & $\mathrm{n}$ & $\mathrm{Q}_{\mathrm{o}}\left(\mathrm{mg} \mathrm{g}^{-1}\right)$ & $\mathrm{b}\left(\mathrm{L} \mathrm{mg}^{-1}\right)$ & $\mathrm{R}_{\mathrm{L}}$ \\
\hline $\mathrm{Cu}$ & 15,07 & 4,58 & 41,64 & 0,210 & 0,0093 \\
$\mathrm{Zn}$ & 11,28 & 4,37 & 37,59 & 0,079 & 0,0238 \\
$\mathrm{Cd}$ & 19,33 & 6,86 & 48,33 & 0,0366 & 0,0310 \\
\hline
\end{tabular}

A constante de Langmuir $\mathrm{Q}_{0}$ e a constante $\mathrm{K}_{\mathrm{f}}$ de Freundlich, as quais estão relacionadas com a capacidade de adsorção máxima, indicaram a seguinte seqüência de seletividade para a zeólita ZB/100: $\mathrm{Cd}>\mathrm{Cu}>\mathrm{Zn}$. Os valores das constantes $\mathrm{R}_{\mathrm{L}}$ e $\mathrm{n}$ para todos os metais mostraram que a adsorção foi um processo completamente favorável na faixa de concentração estudada.

A eficiência de remoção dos metais de amostras de efluentes de galvanoplastia usando-se a zeólita ZB/100 mostrou-se efetiva (Tabela 4). As concentrações dos metais após o tratamento com a zeólita estavam dentro do limite máximo permissível em efluentes segundo a Resolução CONAMA no $20^{18}$.

\section{CONCLUSÕES}

É possível utilizar as zeólitas sintetizadas com as cinzas residuárias de carvão como eficientes trocadoras de íons para a remoção de cádmio, zinco e cobre de soluções aquosas.

A zeólita preparada a partir da cinza da base da chaminé apresentou a maior eficiência de retenção para os íons metálicos em relação àquelas preparadas com a cinza da Tremonha e com a cinza do a)

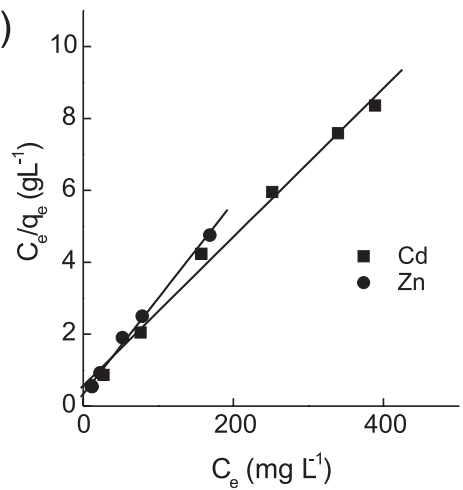

b)

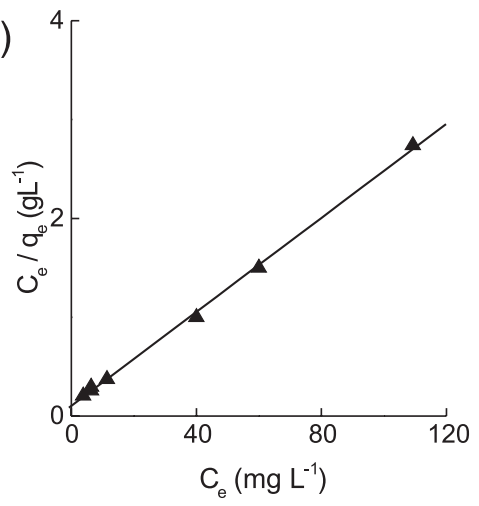

Figura 5. Isoterma de adsorção de Langmuir para (a) $\mathrm{Cd}^{2+}, \mathrm{Zn}^{2+}$; (b) $\mathrm{Cu}^{2+}$ $\left(1 \mathrm{~g}\right.$ zeólita, $\left.\mathrm{T}=25^{\circ} \mathrm{C}\right)$

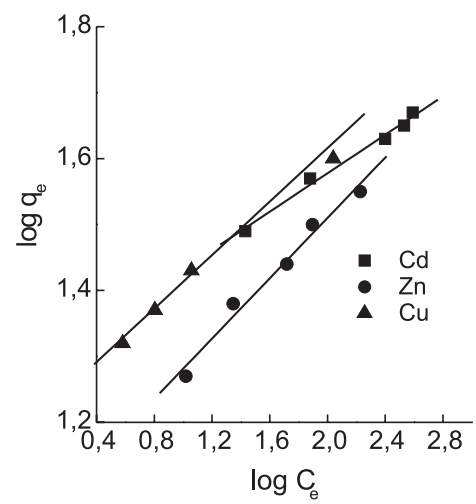

Figura 6. Isoterma de adsorção de Freundlich para o $\mathrm{Cd}^{2+}, \mathrm{Zn}^{2+}$ e Cu$u^{2+}(1 \mathrm{~g}$ zeólita, $T=25^{\circ} \mathrm{C}$ )

Tabela 4. Concentração $\left(\mathrm{mg} \mathrm{L}^{-1}\right)$ de metais em amostras de efluentes de galvanoplastia antes $\left(\mathrm{C}_{\mathrm{i}}\right)$ e após contato $\left(\mathrm{C}_{\mathrm{f}}\right)$ com a zeólita $\mathrm{ZB} /$ $100(*)$

\begin{tabular}{lllll} 
Metal & \multicolumn{2}{c}{ Amostra 1 } & \multicolumn{2}{c}{ Amostra 2 } \\
\hline & $\mathrm{C}_{\mathrm{i}}$ & $\mathrm{C}_{\mathrm{f}}$ & $\mathrm{C}_{\mathrm{i}}$ & $\mathrm{C}_{\mathrm{f}}$ \\
\cline { 2 - 5 } $\mathrm{Zn}$ & 33,0 & 0,368 & 74,6 & 5,06 \\
$\mathrm{Cd}$ & 0,548 & 0 & 0,358 & 0 \\
$\mathrm{~Pb}$ & 2,25 & 0,530 & - & - \\
$\mathrm{Cu}$ & 21,6 & 0 & - & -
\end{tabular}

(*) massa da zeólita $=1 \mathrm{~g}$; volume da solução $=100 \mathrm{~mL} ; \mathrm{T}=25^{\circ} \mathrm{C}$; tempo de agitação $=24 \mathrm{~h}$ 
Malachador. Quanto mais fina era a partícula da zeólita, maior foi a capacidade de troca iônica.

A zeólita da cinza da base da chaminé mostrou uma eficiência de remoção $\geq 70 \%$ para os íons cádmio, zinco e cobre após $2 \mathrm{~h}$ de agitação, revelando o grande potencial para o seu uso como adsorvedor de baixo custo no tratamento de efluentes contaminados com níveis altos de metais.

\section{AGRADECIMENTOS}

Os autores agradecem o apoio financeiro do CNPq.

\section{REFERÊNCIAS}

1. Lin, C-F.; Hsi, H-C.; Environ. Sci. Technol. 1995, 29,1109.

2. Singer, A.; Berkgaut, V.; Environ. Sci. Technol. 1995, 29, 1748.

3. Amrhein, C.; Haghnia, G. H.; Kim, T. S.; Mosher, P. A.; Gagajena, R. C.; Amanios, T.; Torre, L.; Environ. Sci. Technol. 1996, 30, 735.

4. Smith, J. A.; Zeolites 1984, 4, 309.
5. Breck, D. W.; Zelolite Molecular Sieve, John Wiley \& Sons: New York, 1984.

6. Smart, L.; Moore, E.; Solid State Chemistry. An introduction, Chapamn \& Hall: London, 1992.

7. Keraoui-Ouki, S.; Cheeseman, C. R.; Perry, R.; J. Chem. Technol. Biotechnol. 1994, 59, 121.

8. Pansini, M.; Miner. Deposita 1996, 31, 563.

9. Shih, W-H.; Chang, H-L.; Mater. Lett. 1996, 28, 263.

10. Steenbruggen, G.; Hollman, G. G.; J. Geochem. Explor. 1998, 62, 305.

11. Henmi, T.; Clay Sci. 1987, 6, 277.

12. Nota Técnica sobre Tecnologia de Controle - NT 16, Galvanoplastias, CETESB, 1990.

13. Ouki, S. K.; Kavannah, M.; Wat. Sci. Technol. 1999, 39, 115.

14. Semmens, M. J.; Seyfarth, M. Em Natural Zeolites Occurrence, Properties, Use; Sand, L. B.; Mumpton, J., eds.; Pergamon Press: New York, 1978.

15. Faust; S. D.; Aly, O. M.; Adsorption Process for Water Treatment, Butterworths: London, 1987.

16. Perrich, J. R.; Actived Carbon Adsorption for Waste Water Treatment, CRC Press: Boca Raton, 1981.

17. Weber, T. W.; Chakravo, R. K.; AIChE J. 1974, 20, 228.

18. Ministério do Desenvolvimento Urbano e Meio Ambiente. Resolução CONAMA n 20 (art. 21) de 18 de junho de 1986. D.O.U. Executivo, 11356, 30/07/1986. 